\title{
Inhalt, Vol. 11, No. 6, 1988
}

\section{Contents}

Impressum 248

Schmidt, F. (Mannheim)

Gefahren durch Rauchen und Passivrauchen 250

Kleeberg, U. R. (Hamburg)

Ätiologie des Melanoms 254

Ammon, A.; Eiffert, H.; Weber, M. H.; Rummel, J.;

Níemann, J. (Göttingen)

Tumormarker bei dialysepflichtiger Niereninsuffizienz. Ein Vergleich der Antigene MCA, Ca 15-3, CA125, CA19-9 und CEA . 260

Hirsch, F. W.; Kraaz, B.; Loöhr, G. W.; Bross, K. J. (Freiburg) Wirksamkeit und Nebenwirkungen von rekombiniertem Interferon alpha-2a bei Patienten mit metastasierendem Hypernephrom 263

Informationen für die Klinik 266

Holtkamp, W.; Nagel, G. A. (Göttingen)

Stimulation der Prolaktinsekretion mit Thyreoliberin (TRH).

In-vivo- und In-vitro-Untersuchungen beim metastasierenden

Mammakarzinom 267

Hinweise für Autoren 272

Paschke, R.; Worst, P.; Brust, J.; Queißer, W. (Mannheim)

Hepatotoxizität bei Etoposid-Ifosfarnid-Kombinations-Cherno-

therapie 273

Radtke, H.; Pees, H. W.; Schwambom, J.; Thumser, B.;

Daus, H. (Homburg/Saar)

Sequentielle hochdosierte Cytarabin-Therapie in Kombination mit Asparaginase bei der akuten myeloischen Leukämie 276

Kurzmitteilungen

Larson, D. A.; Bottles, K.; Federle, M.; Fippin, L.; Luce, J.

(San Francisco, California/USA)

Skelettmuskel-Metastasen bei Bauchspeicheldrüsenkrebs .... 282

Nagel, G. A.; Beyer, J.-H.; Holtkamp, W.; Emrich, D.;

Kneba, M.; Luig, H.; Marschner, N.; Rauschning, W.;

linger, C; Wander, H. E. (Göttingen)

Hochdosiertes Epirubicin + Cyclophosphamid (HD-EC) beim

metastasierenden Mammakarzinom: eine Dosisfindungs-Studie . 287

Nagel, G. A.; Rauschning, W.; Ammon, A.; Beyer, J.-H.;

Emrich, D.; Holtkamp, W.; Kneba, M.; Luig, H.; 
Marschner, N.; Unger, C. (Göttingen)

Hochdosiertes Epirubicin + Cyclophosphamid (HD-EC) beim

metastasierenden Mammakarzinom: Daten einer Phase-II-

Studie 289

Kreis, W.; Budman, D. R.; Delli Bovi, P.;

Vinciguerra, V. (Manhasset, N.Y./USA)

Rascher Anstieg von Serum-Testosteron nach Beendigung einer

Langzeitbehandlung beim Prostatakarzinom mit einem LHRH-

Agonist 292

Sonderbände 293

Kurzmitteilungen

Unger, C; Eibl, H.; B reiser, A.; von Hey den, H. W.; Nagel, G. A. (Göttingen); Engel, J.;

Hilgard, P.; Sindermann, H.; Peukert, M. (Bielefeld)

Hexadecylphosphocholin (D18506) bei der topischen Behand

lung von Hautmetastasen: Eine Phase-I-Studie 295

Autorenverzeichnis $1988 \quad 297$

Jahres-Inhalt $1988 \quad 299$

Imprint248

Schmidt, F. (Mannheim)

Health Risks by Smoking and Passive Smoking $\quad 250$

Kleeberg, U. R. (Hamburg)

Etiology of Melanoma 254

Ammon, A.; Eiffert, H.; Weber, M. H.; Rummel, J.;

Niemann, J. (Göttingen)

Tumor Markers in Patients with Chronic Renal Failure Treated

by Hemodialvsis - A Comparison of the Antigens MCA, CA 15-3,

CA125, CAÍ9-9, andCEA 260

Hirsch, F. W.; Kraaz, B.; Loöhr, G. W.; Bross, K. J. (Freiburg)

Interferon alpha-2a in the Treatment of Patients with Advanced

Renal Cell Carcinoma - Effects and Side Effects 263

Clinical Information 266

Holtkamp, W.; Nagel, G. A. (Göttingen)

Stimulation of Plasma-Prolactin by Thyrotropin-Releasing-

Hormone in Patients with Metastatic Breast Cancer. In vivo and

in vitro Studies $\quad 267$

Instructions to Authors 272

Paschke, R.; Worst, P.; Brust, J.; Queißer, W. (Mannheim)

Hepatotoxicity of Etoposide/Ifosfamide Combination Chemo

therapy 273

Radtke, H.; Pees, H. W.; Schwambom, J.; Thumser, B.;

Daus, H. (Homburg/Saar)

Sequential High-Dose Cytosine Arabinoside in Combination

with Asparaginase in Acute Non-lymphocytic Leukemia 276

Short Communications

Larson, D. A.; Bottles, K.; Federle, M.; Fippin, L.;

Luce, J. (San Francisco, California/USA) 
Skeletal Muscle Metastases in Pancreatic Cancer 282

Nagel, G. A.; Beyer, J.-H.; Holtkamp, W.; Emrich, D.; Kneba, M.; Luig, H.; Marschner, N.;

Rauschning, W.; Unger, C; Wander, H. E. (Göttingen)

High-Dose Epirubicin + Cyclophosphamide (HD-EC) in Metas

tatic Breast Cancer: A Dose-Finding Study 287

Nagel, G. A.; Rauschning, W.; Ammon, A.; Beyer, J.-H.; Emrich, D.; Holtkamp, W.; Kneba, M.;

Luig, H.; Marschner, N.; Unger, C. (Göttingen)

High-Dose Epirubicin + Cyclophosphamide (HD-EC) in Metastatic Breast Cancer:

Preliminary/Data of a Phase-II Study .... 289

Kreis, W.; Budman, D. R.; Delli Bovi, P.;

Vinciguerra, V. (Manhasset, N.Y./USA)

Rapid Rise of Serum Testosterone Following Discontinuation of

Long Term Treatment of Prostate Carcinoma with an LHRH-

Agonist 292

Special Editions 293

Short Communications

Unger, C; Eibl, H,; Breiser, A.; von Hey den, H. W.;

Nagel, G. A. (Göttingen); Engel, J.; Hilgard, P.;

Sindermann, H,; Peukert, M. (Bielefeld)

Hexadecylphosphocholine (D18506) in the Topical Treatment of

Skin Metastases: A Phase-I Trial 295

Author Index 1988297

Complete Contents $1988 \quad 299$

Bibliographischer Hinweis: Inhaltsverzeichnisse dieser Zeitschrift erscheinen regelmäßig in current contents ${ }^{\circledR}$ sowie in anderen bibliographischen Diensten. 